inhibiting SMAD3, PTEN, ATM, IL18 and BCL2. Consequentially, the observed network-wide changes on LDHC silencing are predicted to negatively influence cellular growth and proliferation, cell migration and cell infiltration. The LDHC-associated network indicated a higher-level regulation by $\operatorname{miR} 378 \mathrm{a}-3 \mathrm{p} \quad(\mathrm{p}=1.4 \mathrm{e}-7, \mathrm{z}=-3.117)$, affecting the downstream mechanistic in LDHC-expressing cells. Interestingly, the miR378a causal network also indicated inhibition of the immune response in LDHC-positive cells. TIDE analysis indicated that high expression of LDHC in the METABRIC Her2 breast cancer cohort (TIDE score $=1.97, p=0.049$ ), and to a lesser extent in triple negative breast cancer (TIDE score $=0.466, \mathrm{p}=0.642$ ), decreases the beneficial effect between CTLs and overall survival observed in LDHC Low tumors. Concurrently, LDHC-silenced cells displayed a proinflammatory gene expression and cytokine profile and down-regulated the expression of PD-L1 and Gal-9 immune checkpoints.

Conclusions Our findings provide an indication of potential CTL dysfunction in breast tumors with high LDHC expression and suggests that therapeutic targeting of LDHC may inhibit tumor growth while releasing the anti-tumor immune response in breast cancer.

Disclosure Information A. Naik: None. J. Decock: None.

\section{P06.05 ENDOGENOUS T-CELL RESPONSES TO TEN MAJOR CANCER TESTIS ANTIGENS ARE FREQUENT IN ESOPHAGO-GASTRIC ADENOCARCINOMA AND ANTIGEN-SPECIFIC T CELLS CAN BE EXPANDED USING CD40-ACTIVATED B CELLS}

${ }^{1} \mathrm{M}$ Thelen*, 'M Garcia-Marquez, ' IJ Lehmann, 'D Keller, 'E Preugszat, ${ }^{2,3} \mathrm{M}$ von BergweltBaildon, 'HA Schlößer. 'Center for Molecular Medicine Cologne, Cologne, Germany; 2Department of Internal Medicine III, University Hospital, Ludwig Maximilians University, Munich, Germany; ${ }^{3}$ German Cancer Consortium (DKTK), Heidelberg, Germany

\subsection{6/jitc-2021-ITOC8.39}

Background Tumor-associated antigens (TAAs) and especially cancer testis antigens (CTAs) are classical tumor-specific targets for immunotherapies. As TAAs are shared between patients, strategies aiming to exploit these targets are scalable and potentially applicable across different types of cancer. Loss of target antigens and other mechanisms of immune escape have limited the success of CTA-directed immunotherapy. CAR T cells and other highly effective cellular therapies have renewed the interest in TAAs. Especially combined targeting of multiple antigens appears highly promising as recently shown in lymphoma. In our study, we aimed to characterize CTA-expression patterns and their impact on endogenous T-cell responses, T-cell abundance and antigen-presentation in esophago-gastric adenocarcinoma (EGA).

Materials and Methods 41 treatment-naïve EGA patients were included in our study. RNA of tumor and patient-matched healthy tissue was isolated and used for NanoString based RNA expression analysis of 26 CTAs and 25 genes associated with antigen-presentation. Based on CTA expression, 10 peptide pools were selected and co-cultured with peripheral blood mononuclear cells (PBMCs, $n=21$ ) to determine cellular anti-tumor immune responses in a FluoroSpot assay. Tcell abundance was assessed using immunohistochemistry (CD3, CD8) and digital image analyses of tumor area and invasive margin. Autologous CD40 activated B cells were used to expand antigen-specific T cells using peptide pools of CTAs.

Results NanoString analysis revealed pronounced differences regarding CTA expression, with CEP55 and MAGEA3/6 showing strong expression, while NY-ESO-1 or MAGEA1 were only weakly expressed. $68.3 \%$ (28/41) of the patients showed expression of $\geq 5 / 10$ analyzed TAAs simultaneously. In line with the frequent expression, $75.0 \%$ of the patients showed a cellular response against at least one of the TAAs. T-cell responses were most frequently detected to Survivin and NY-ESO-1 $(65.0 \%$ and $52.6 \%$ of patients, respectively), while only $20.0 \%$ responded to CEP55 or TTK peptide pools. Overall, 6/20 patients showed cellular responses against $\geq 5$ TAAs simultaneously. We found a strong correlation of T-cell abundance and antigen-presentation. In addition, patients with a high Immune-Score showed increased TAA expression. Finally, we demonstrate feasibility of TAAspecific T-cell expansion using CD40 activated B cells as potential strategy to induce or enhance TAA immune responses in EGA.

Conclusions Our study highlights the importance of TAAs in EGA. The identified antigens are highly relevant for immunomonitoring of clinical trials and as targets for immunotherapy. Personalized immunotherapeutic strategies targeting EGA-specific or even patient specific TAAs appear highly promising in this challenging disease.

Disclosure Information M. Thelen: None. M. Garcia-Marquez: None. J. Lehmann: None. D. Keller: None. E. Preugszat: None. M. von Bergwelt-Baildon: B. Research Grant (principal investigator, collaborator or consultant and pending grants as well as grants already received); Modest; Astellas, Roche, MSD. D. Speakers Bureau/Honoraria (speakers bureau, symposia, and expert witness); Modest; BMS. F. Consultant/Advisory Board; Modest; BMS. H.A. Schlößer: B. Research Grant (principal investigator, collaborator or consultant and pending grants as well as grants already received); Significant; Astra Zeneca.

\section{P06.06 ENHANCING TRAFFICKING AND RESISTANCE TO IMMUNOSUPPRESSION OF SYNTHETIC AGONISTIC RECEPTOR-TRANSDUCED T CELLS IN SOLID TUMOR MODELS}

${ }^{1,2} \mathrm{M}$ Schwerdtfeger*, ${ }^{1} \mathrm{M}$ Benmebarek, ${ }^{1} \mathrm{~F}$ Märkl, ${ }^{1} \mathrm{CH}$ Karches, ${ }^{1} \mathrm{~A}$ Öner, ${ }^{3} \mathrm{M}$ Geiger, ${ }^{1} \mathrm{~B}$ Cadilha, ${ }^{1} \mathrm{~S}$ Endres, ${ }^{2} \mathrm{~V}$ Desiderio, ${ }^{3} \mathrm{C}$ Klein, ${ }^{1,4,5} \mathrm{~S}$ Kobold. ${ }^{1}$ Center of Integrated Protein Science Munich (CIPS-M) and Division of Clinical Pharmacology, Department of Medicine IV, Klinikum der Universität München, LMU Munich, Member of the German Center for Lung Research (DZL), Munich, Germany; ${ }^{2}$ Department of Experimental Medicine, University of Campania 'Luigi Vanvitelli', Naples, Italy; ${ }^{3}$ Roche Innovation Center Zurich, Schlieren, Switzerland; ${ }^{4}$ German Center for Translational Cancer Research (DKTK), partner site Munich, Munich, Germany; ${ }^{5}$ Einheit für Klinische Pharmakologie (EKLiP), Helmholtz Zentrum München, German Research Center for Environmental Health (HMGU), Munich, Germany

\subsection{6/jitc-2021-ITOC8.40}

Background Chimeric antigen receptor therapy - although very efficacious in B cell malignancies - is facing many challenges which limit its success in solid tumors, e.g. on-target off-tumor toxicities, antigen heterogeneity, lack of $\mathrm{T}$ cell migration into tumors and an immunosuppressive tumor microenvironment. To better control on-target off-tumor effects and address antigen heterogeneity we developed a modular approach where we equipped $\mathrm{T}$ cells with a synthetic agonistic receptor (SAR). The SAR is only activated in the 